\title{
A New Proxy Application for Compressible Multiphase Turbulent Flows
}

\author{
Tania Banerjee ${ }^{1}$, Jason Hackl², Mrugesh Sringarpure ${ }^{2}$, Tanzima Islam², \\ S. Balachandar ${ }^{2}$, Thomas Jackson ${ }^{2}$ and Sanjay Ranka ${ }^{1}$ \\ ${ }^{1}$ Department of Computer Information Science and Engineering, University of Florida \\ ${ }^{2}$ Department of Mechanical and Aerospace Engineering, University of Florida \\ ${ }^{3}$ Lawrence Livermore National Laboratory \\ ${ }^{3}$ islam3@llnl.gov \\ 1,2\{tmishra, jason.hackl,mrugeshs, balals, tlj,ranka\}@ufl.edu
}

\begin{abstract}
CMT-nek is a simulation code for performing high fidelity, predictive simulations of particle laden explosively dispersed turbulent flows under conditions of extreme pressure and temperature. CMT has many applications including lithotripsy, dust explosions in coal mines, and explosive detonation. The physical processes underlying these applications are complex and cover a very wide range of spatial and temporal scales.

The underlying workflow of this application requires a very rich set of computation on multiple types of data structures and data access patterns. Additionally, the simulation of such codes require high spatial and temporal resolution resulting in large memory footprint and enormous computing power that is only available on extant petascale and exascale supercomputers. The workflow and the underlying patterns are representative of other scientific applications and hence have the potential to serve as an important benchmark for next generation supercomputers.

In this paper $^{1}$, we describe CMT-bone that serves as a proxy-app for CMT-nek. Proxy applications provide a simplified framework for computational scientists and system designers to investigate new node architectures, programming approaches, algorithmic design choices and optimization techniques. CMTbone retains the workflow of CMT-nek and encapsulates key data structures, computational and communication patterns of CMTnek. It reduces the number of variables used and corresponding compute and communication operations performed at each step of the work.
\end{abstract}

Validation of CMT-bone with the CMT-nek using the VERITAS tool developed at LLNL shows that CMT-nek is a good proxy. Such a validation is important as it shows that important computational charateristics of CMT-nek are embedded in CMTbone and any experimental performance analysis of the latter will be representative of the former.

\section{INTRODUCTION}

CMT-nek is a simulation code for performing high fidelity, predictive simulations of particle laden explosively dispersed turbulent flows under conditions of extreme pressure and temperature. Such flows are predominant in many medical, ecological and national security applications, such as lithotripsy, dust explosions in coal mines, and explosive detonation. Being able to accurately predict such flows is crucial as it enhances our ability to control such flows and

\footnotetext{
${ }^{1}$ A preliminary version of this work will appear in Tania Banerjee, Jason Hackl, Mrugesh Sringarpure, Tanzima Islam, Siva Balachandar, Thomas Jackson and Sanjay Ranka, CMT-bone: A Proxy Application for Compressible Multiphase Turbulent Flows, Proceedings of HiPC 2016.
}

develop pathways for understanding particle dispersal. CMTnek simulates multiphase flows as an Eulerian phase obeying the Euler equations of gas dynamics at the macroscale, twoway coupled with Lagrangian point particles whose forces (on and from the carrier gas) are modeled at the microscale. Governing equations for both phases are discretized in space and time and solved numerically.

CMT-nek reuses the basic setup and initialization routines of Nek5000 [1] which was developed at Argonne National Laboratory by Fischer et al. Nek5000 supports simulation of unsteady and incompressible fluid flow with thermal and passive scalar transport. The code is open source and is highly scalable with strong scaling runs demonstrated over a million MPI ranks on ALCF BG/Q Mira[2]. However, Nek5000 may be used for low speed flows only as a result of its formulation and discretization strategies. CMT-nek uses improved physicsinformed models and closures for compressible multiphase turbulence. At the same time, the application CMT-nek is compute intensive and is expected to be deployed over petascale and exascale supercomputers for improved performance. The computation and data access patterns of CMT-nek are representative of complex scientific applications. In fact, CMTnek could soon be an important benchmark for evaluating performance, energy and temperature characteristics of supercomputers in the near future. Some basic features of CMT-nek are described as follows.

1) Domain decomposition: This is an important step to achieve parallelism in the program. CMT-nek uses discontinuous Galerkin methods for domain decomposition, which makes it an interesting case study for communication scaling across homogeneous and heterogeneous platforms. The primary communication pattern is nearest neighbor point to point communication to exchange migrating particles and surface fluxes. Surface flux exchanges happen between a maximum of 26 processes holding the 26 neighbors to an element. A spectral element in CMT-nek is the smallest unit that is distributed across processors and an element cannot be further subdivided as part of the domain decomposition algorithm in CMT-nek.

2) Unique computation structures: There are a number of computations done in CMT-nek, such as derivative 
computations and dealiasing, that rely on small dense matrix-matrix multiplications for three dimensional spectral elements. The uniqueness of these matrix-matrix multiplication is that depending on the spatial direction for computation $(x, y$ or $z$ ), the data is accessed from memory either in unit strides, or in strides of $N$ and $N^{2}$, where $N$ is the number of grid points in a cubic spectral element along any one direction. Some of the other computation patterns are dot-products, vector updates and matrix-vector multiplications. In general, the computation to data access ratio is low in CMT-nek. This is also a common characteristic of many other scientific applications and makes CMT-nek a good representative of such applications.

3) Coupled data structures: The multiphase property, or presence of particles in the fluid flow, requires coupled data structures for the simulation to capture interactions between particles and fluid. The interaction results in updated particle locations. For each particle, CMT-nek computes its velocity and force fields (comprising of drag, stress gradient, inviscid, and viscous components) at the respective particle location. Thus, it involves extensive computation. Also, moving particles may create highly non-uniform assignment of particles to elements resulting in challenging load balancing issues. Point particle tracking results in a set of challenging problems for efficient computation, memory access patterns, communication, and load balancing.

4) Variable compute intensiveness: A single time step in the simulation of CMT-nek uses various computer resources, such as CPU, memory, and the network. However, different portions of code use these resources differently which makes it a challenging application to study multiobjective optimization problems involving performance, energy and power consumption. Such applications and matrix studies are expected to be very important for next generation supercomputers.

Based on the above chararcterisitcs, CMT-nek has the potential to become an important application to evaluate current and future architectures.

In this paper we describe the development of CMT-bone which is a proxy application of CMT-nek, and in the process also describe the basic computation and communication structures of CMT-nek. Proxy applications are small, portable codes that represent the important stages of the parent application in terms of performance, power, and energy analysis. Proxy applications are thus easier to test, simulate and optimize compared to the parent applications, and are important vehicles for hardware/software co-design. Validation is an equally important step and is needed to ensure that the important kernels are represented correctly, so that experimental or other analysis performed on the proxy application is representative of the parent application. Section VII presents some examples of large scientific codes that have been optimized using proxy applications.

The two main contributions of this paper are as follows:

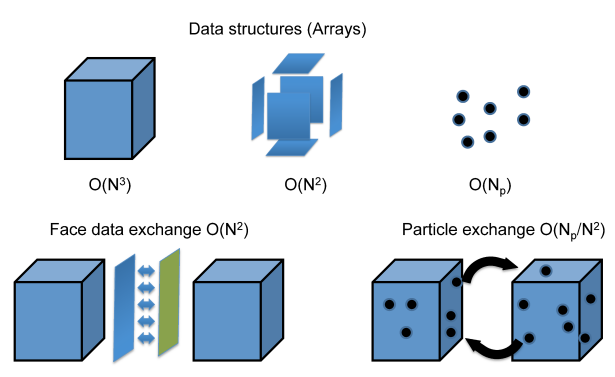

Fig. 1: Key data structures and communication operations in CMT-nek

1) A proxy application was developed for a complex code simulating compressible multiphase turbulent flows. CMT-bone involves different data structures as it has to store volume data, surface data and particle data, and employs different memory access patterns on these data types. Hence, CMT-bone is an useful application that can help us evaluate different architectures, communication networks, computation, and scalability issues. The particle simulation capabilities of CMT-bone allow us to experiment with different dynamic load balancing strategies for particle tracking.

2) We demonstrate the proxy app validation procedure using VERITAS [3] for specific kernels. The technique is based on using data collected by hardware performance counters. The performance counters available on an architecture are then cateogorized into resource groups. Bottlenecks in scaling the parent and proxy application are then used along with machine learning techniques for validation.

The rest of the paper is described as follows. In Section II, we describe our target application CMT-nek and its implementation details. Section III presents the governing equations for CMT-nek computations, while Section IV presents the implementation details of CMT-bone. The experimental results are presented in Section V which validates CMT-bone as a proxy app for CMT-nek. Finally, conclusions are given in Section VIII.

\section{CMT-NEK}

CMT-nek extends Nek5000 for solving the compressible Navier-Stokes equations for multiphase flows. Under conditions of extreme pressure and temperature, CMT-nek is able to perform high fidelity, predictive simulations of particle laden explosively dispersed turbulent flows. The three-dimensional Euler equations corresponding to the gas dynamics can be described as a conservation law (Equation (1), Section on Governing Equations). These equations are reduced to a system of ordinary differential equations for the conserved variables $U$ at each of $N_{x} \times N_{y} \times N_{z}$ quadrature nodes on each of $N_{e l}$ finite elements via the discontinuous Galerkin spectral element method. The end result appears in semi-discrete form of Equation (38). The two terms on the right hand side of Equation (38) must be evaluated on each element. The first 
TABLE I: Storage of volume data in CMT-nek.

\begin{tabular}{ccc}
\hline Variable & Dimension & Physical quantity \\
vtrans $(\ldots, 1)$ & $N_{x}, N_{y}, N_{z}, N_{e l}, 1$ & gas density $\rho_{g}$ \\
vtrans $(\ldots, 2)$ & $N_{x}, N_{y}, N_{z}, N_{e l}, 1$ & constant-pressure specific heat $c_{p g}$ \\
$\operatorname{vtrans}(\ldots, 3)$ & $N_{x}, N_{y}, N_{z}, N_{e l}, 1$ & constant-volume specific heat $c_{v g}$ \\
t $(\ldots, 1)$ & $N_{x}, N_{y}, N_{z}, N_{e l}, 1$ & temperature $T_{g}$ \\
pr & $N_{x}, N_{y}, N_{z}, N_{e l}$ & pressure $p_{g}$ \\
vx, vy and vz & $N_{x}, N_{y}, N_{z}, N_{e l}$ & velocity components $u_{g}, v_{g}, w_{g}$ \\
U & $N_{x}, N_{y}, N_{z}, 5, N_{e l}$ & conserved unknowns $($ Equation $(2))$ \\
rx & $M_{x}, M_{y}, M_{z}, 9, N_{e l}$ & weighted metrics $\mathcal{M}_{M} \mathcal{I}\left[\frac{1}{J} \frac{d r_{i}}{d x_{j}}\right]$ \\
vxd, vyd and vzd & $M_{x}, M_{y}, M_{z}, N_{e l}$ & $\mathcal{I} \mathbf{u}, \mathcal{I} \mathbf{v}, \mathcal{I} \mathbf{w}$ \\
\hline
\end{tabular}

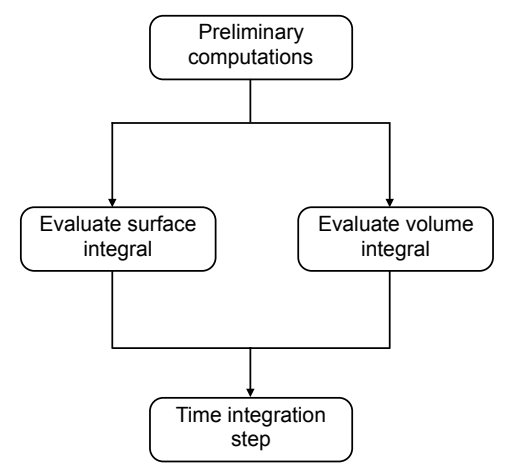

term contributes to $\partial \mathbf{U} / \partial t$ at every quadrature point on the grid, and the second term is an additional effect added only to quadrature points lying on element faces.

The key data structures, communication and computation structures in CMT-nek are described in Figure 1. A large fraction of the variables used for the evaluation of the corresponding equations require the use of multidimensional arrays. The key variables required correspond to volume and surface data for the flow.

Being implemented in Fortran 77, CMT-nek predominantly uses multidimensional column-major arrays for storing volume and surface data. Table I lists array names, their dimensions, and the corresponding physical quantities they represent. $N_{x}, N_{y}, N_{z}$ are the number of GLL quadrature nodes in each direction of a given element $\Omega_{e}$ in Nek5000. $N_{e l}$ is the number of elements for a given MPI rank. vtrans and $\mathrm{t}$ are arrays of rank five - the first four dimensions store volume data for all elements on a given MPI rank, and the last dimension corresponds to one of ldimt passive scalars. The scalar corresponds to values such as gas density, specific heat or temperature (Table I). These have been re-purposed as thermodynamic quantities for our code (Equations (8),(9)).

For each of the faces in an element, surface data is stored contiguously using rank-4 arrays with dimensions $\left(N_{x}, N_{z}, N_{\text {faces }}, N_{e l}\right)$. Here $N_{\text {faces }}$ is the number of bounding surfaces $(6$ for $3 D$ ) or edges ( 4 for $2 D$ ) of an element.

All the point particles are stored in a rank-2 array with dimensions $\left(P t_{p r o p}, N_{p t}\right)$, where $N_{p t}$ is the number of particles owned by an MPI rank and $P t_{\text {prop }}$ is the number of particle properties being tracked. In our code, we track information like particle location $\left(x^{(p)}, y^{(p)}, z^{(p)}\right)$, particle velocity $\left(u_{p}, v_{p}, w_{p}\right)$, fluid velocity at the particle location $\left(u^{(g)}, v^{(g)}, w^{(g)}\right)$ and forces on the particles $\left(F x^{(p)}, F y^{(p)}, F z^{(p)}\right)$. Additional quantities such as heat transfer and particle temperature will also be stored in the future. The number of particle quantities will thus be close to 20 .

The workflow and implementation of CMT-nek can be easily explained by using Equation (38) as a reference. The left-hand side of Equation (38) is explicitly discretized by a third-order total-variation-diminishing Runge-Kutta scheme [4]. This scheme advances the solution of the conservation laws from time $t_{n}$ to $t_{n}+\Delta t$ by computing the terms on right-hand-side of Equation (38) explicitly. Figure 2 shows the workflow of computing these right-hand-side terms. After certain preliminary computations, the surface and volume inte-

gral terms are evaluated and the result is passed on to the time integration step. Each component of this work flow is further expanded to show the most intensive compute operations along with the communication operations in a sequence which represents the actual implementation.

\section{A. Preliminary computations}

A number of computations need to be performed before evaluating surface and volume integrals. These preliminary computations consist of several steps that are shown in Figure (3).

The computation of the surface and volume integral terms on the right-hand-side of Equation (38) require the flux vector $\mathbf{H}$. The latter is a function of conserved variables $\mathbf{U}$ and primitive variables $\rho_{g}, u_{g}, v_{g}, w_{g}, p_{g}$ and $T_{g}$ (see Equations (5),(6),(7)), grid metrics $\partial r_{i} / \partial x_{j}$ and interpolation operator $\mathcal{I}$.

CMT-nek does not treat grids that translate or get deformed during the course of simulation; hence the grid metrics, derivative and interpolation operators remain unchanged. Therefore, these operators are computed once and reused throughout the simulation. The primitive variables $\rho_{g}, u_{g}, v_{g}, w_{g}, T_{g}, p_{g}, a_{g}$, transport properties like $\lambda, \nu, \kappa$ and specific heat values $c_{p g}, c_{v g}$ are computed first. Figure 3 provides the steps for this computation. These computations can be categorized as pointwise arithmetic with $O\left(N^{3}\right)$ computational workload.

The nature and the total number of operations required are dependent on the specific task. For example, the process of computing primitive variables like $\rho_{g}, u_{g}, v_{g}, w_{g}$ involves a single pointwise division operation; the process of computing pressure $p_{g}$ and temperature $T_{g}$ require multiple pointwise addition, subtraction and division. It is important to note that for dealiasing, the velocity components are interpolated onto a fine mesh requiring a matrix-vector multiplication with $O\left(N^{4}\right)$ complexity.

\section{B. Evaluate surface integral}

Once the preliminary computations are done, the next step is evaluation of the right-hand-side term of Equation (38), which represents the surface integral. The flowchart in Figure 4 summarizes the steps for evaluation of a surface integral. 


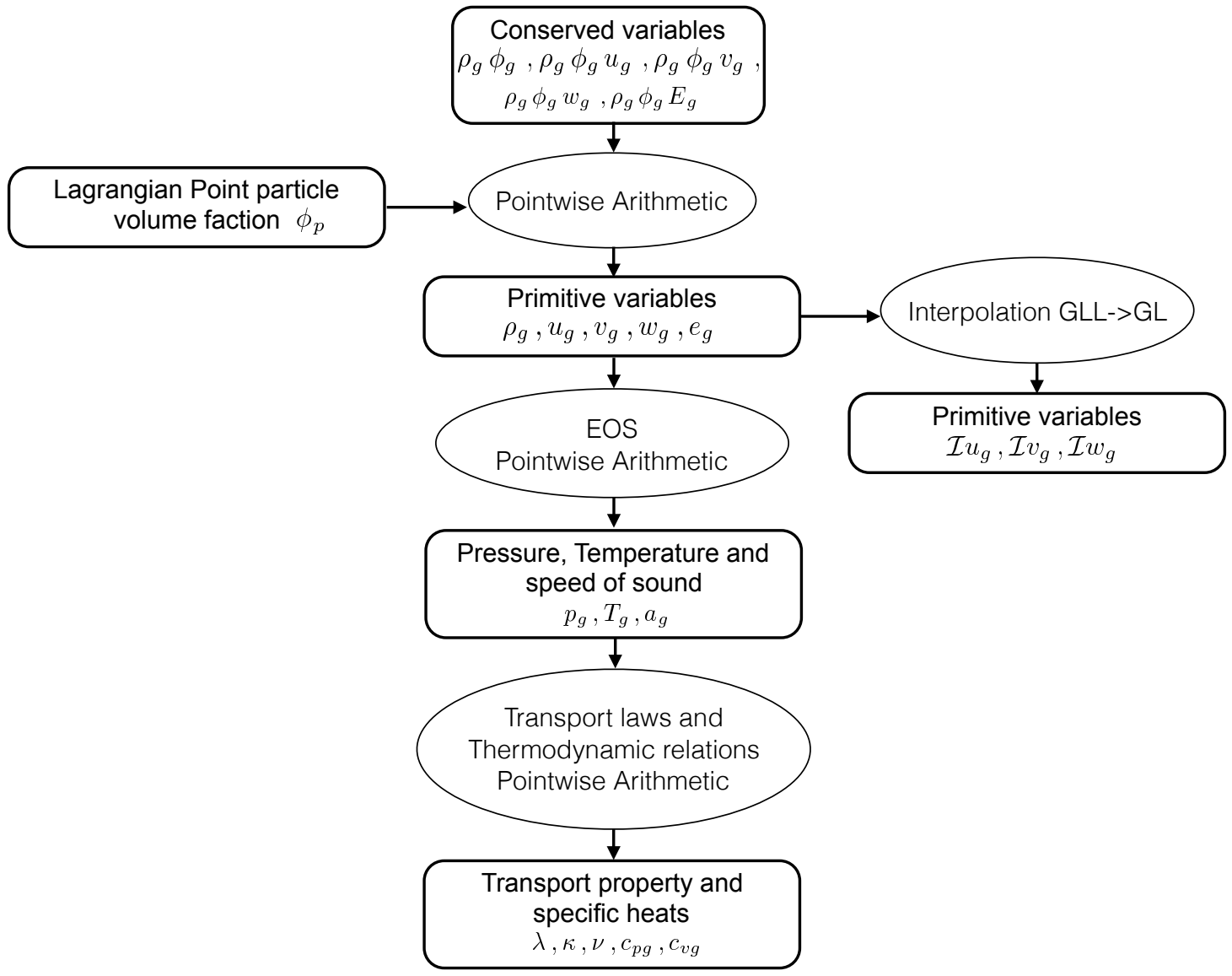

Fig. 3: Workflow of preliminary computations that precedes the evaluate surface and volume integral steps.

For all of the preliminary computations, the primitive variables at each grid point are stored in their respective volume data structure. Unfortunately, this is not the most effective representation for computing properties that require computation on grid points that lie on the surface or for computations that require evaluation of surface integrals. This cost is alleviated by generating a mapping function that takes the surface points (constant strides in a single dimensional storage of a multidimensional array) and maps them into separate but contiguous arrays. This " $f u l l_{-} t o \_f a c e$ " mapping is generated at the time of preprocessing. This step is performed to extract the surface points for all the necessary variables (primitive variables $\rho_{g}, u_{g}, v_{g}, w_{g}, T_{g}, p_{g}, a_{g}$, transport properties $\lambda, \nu, \kappa$ and specific heat values $c_{p g}, c_{v g}$ ). As described above, these are stored in a separate array so that all subsequent steps can be performed efficiently.

The discontinuous Galerkin method allows variables to have jumps (discontinuity) across the element faces, i.e., a gridpoint on any face will have two values associated with two elements that share that face. To support this, the "full_to_face" step provides only the surface data corresponding to all the faces on an element. This information is referred to as "Interior state" in the workflow shown in Figure 4.
The underlying computational structure of Nek5000 corresponds to an unstructured collection of elements. A separate framework is in place that maps the connectivity of all the elements. To determine the surface data corresponding to another element that is proximate to a given element (i.e., the two elements share the same face), this connectivity map has to be used extensively. In practice, this connectivity map is distributed across multiple MPI ranks and has to be suitably decoded. Nek5000 provides a sophisticated domain decomposition and communication framework for this purpose. We refer to this as deriving the "neighboring state" in the workflow. This process of getting the neighboring state is referred to as "GS_op + Pointwise Arithmetic" and requires $O\left(N^{2}\right)$ computational workload.

After the interior and neighboring surface values are derived, the next step is to process the surface fluxes. Thus, for each MPI task, all faces for all elements are processed to interpolate the variables on the surface of an element to a finer grid of dimensions $M_{x} \times M_{z}$ points and the numerical flux $\mathbf{h}^{*}$ is computed at each of these surface nodes. The reader should note the difference between the two flux terms $\mathbf{H}$ and $\mathbf{h}^{*}$ used in this paper. The overall flux function is represented by $\mathbf{H}$. The values of the flux function at the grid points are 


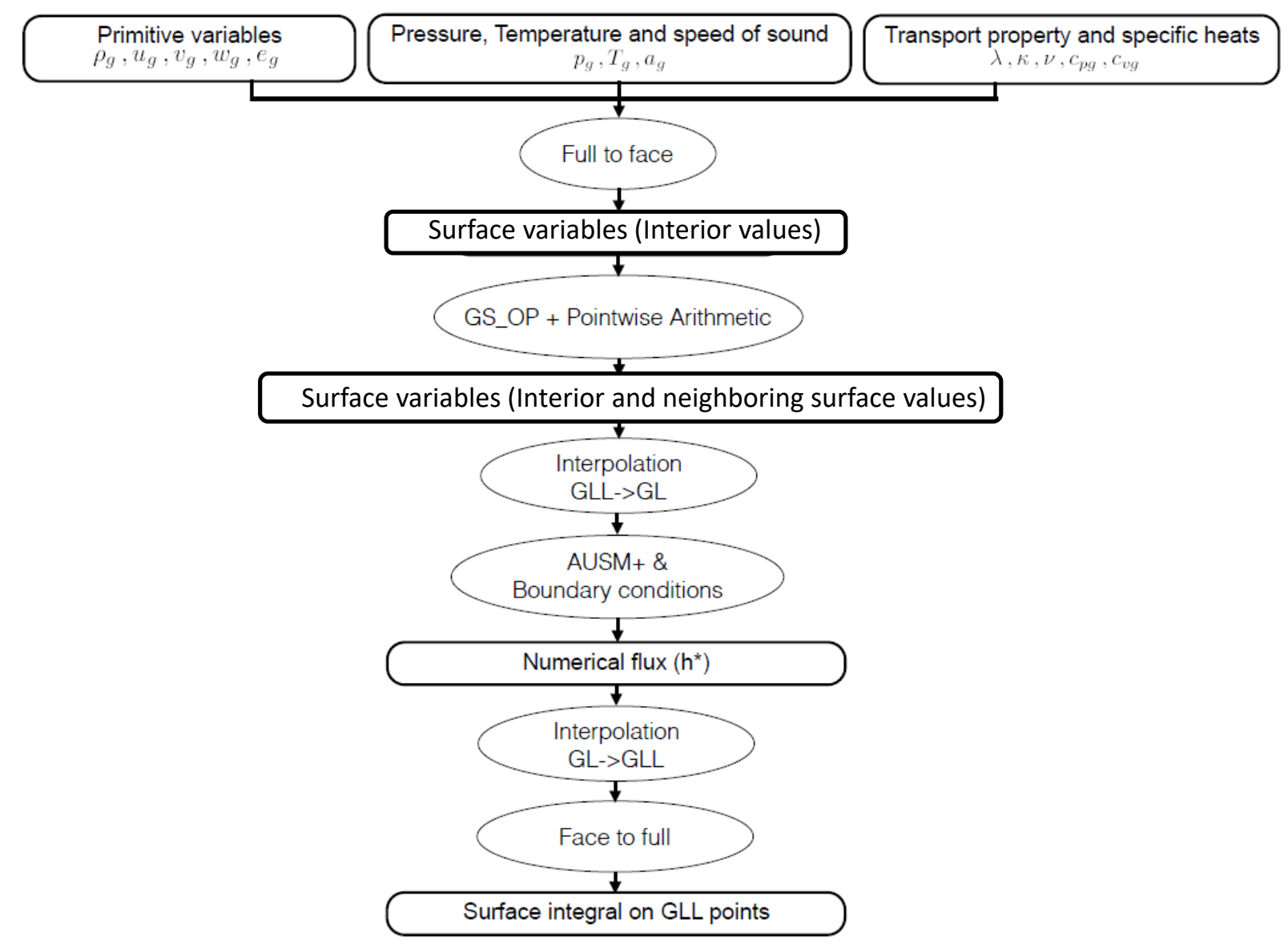

Fig. 4: Workflow of evaluate surface integral step

represented by $\mathbf{h}^{*}$. We use the Advection Upstream Splitting Method (AUSM+)[5] for computing the fluxes numerically. This flux computation can be done for each point on the element faces independently. If a face has no neighbor, a boundary condition is applied instead. Once the fluxes are computed, they get projected back to the $\left(N_{x}, N_{z}\right)$ GLL grid for a given face by tensor-nested matrix multiplication in two successive directions. The total time requirements for this step is $N_{x} \times M_{x}$ matrix $\mathcal{I}_{A}^{\top}$. Once all faces are finished, they get mapped back to the cubic storage by "face_to_full" step.

\section{Evaluate volume integral}

This step evaluates the first term on the right-hand-side of Equation (38) and requires performing computations on cubic data $\left(N_{x}, N_{y}, N_{z}\right)$.

In presence of dealiasing, the conserved variables are interpolated on to the fine grid $\left(M_{x}, M_{y}, M_{z}\right)$ and then combined with the primitive variables. The latter variables are also interpolated on to the fine grid to get the flux vector h. The computation of the flux vector can be classified as "Pointwise Arithmetic" requiring $O\left(N^{3}\right)$ computational effort. To minimize the memory requirements, $\mathbf{h}$ is only stored for one element and one equation at a given time.
The flux vector $\mathbf{h}$ is then multiplied with the corresponding geometric metric vector "rx" and then the transpose of $\mathcal{D}$ is applied for each of the $(r, s, t)$ directions. In presence of dealiasing, this step requires $O\left(M^{4}\right)$ operations. Multiplication by the $N \times M$ matrix $\mathbf{J}^{\top}$ projects the right-hand-side volume-integral term back to the $\left(N_{x}, N_{y}, N_{z}\right)$ grid. The total computational requirements of the interpolation steps is of $O\left(M \times N^{3}\right)$ for $G L L \rightarrow G L$ points and $O\left(N \times M^{3}\right)$ for $G L \rightarrow G L L$ points.

Once the volume integral is computed, it is added to the contribution of the surface integral term to get the right-handside of Equation (38) for all elements and all equations. This combined result is then used by the time integration step. The conserved variables are then advanced to the next time step (stage in RK 3 time integration scheme).

This step is then followed by a particle tracking step. We currently use Lagrangian point particle tracking in the current version of CMT-nek. Currently, the code does not distribute the point particle force data back into Equation (38). In the point particle tracking computations, we first interpolate the fluid velocity at the off-grid point particle location. Using the result of this step, we apply the particle equations of motion (see Equation (12)) and the point particles are moved to their 


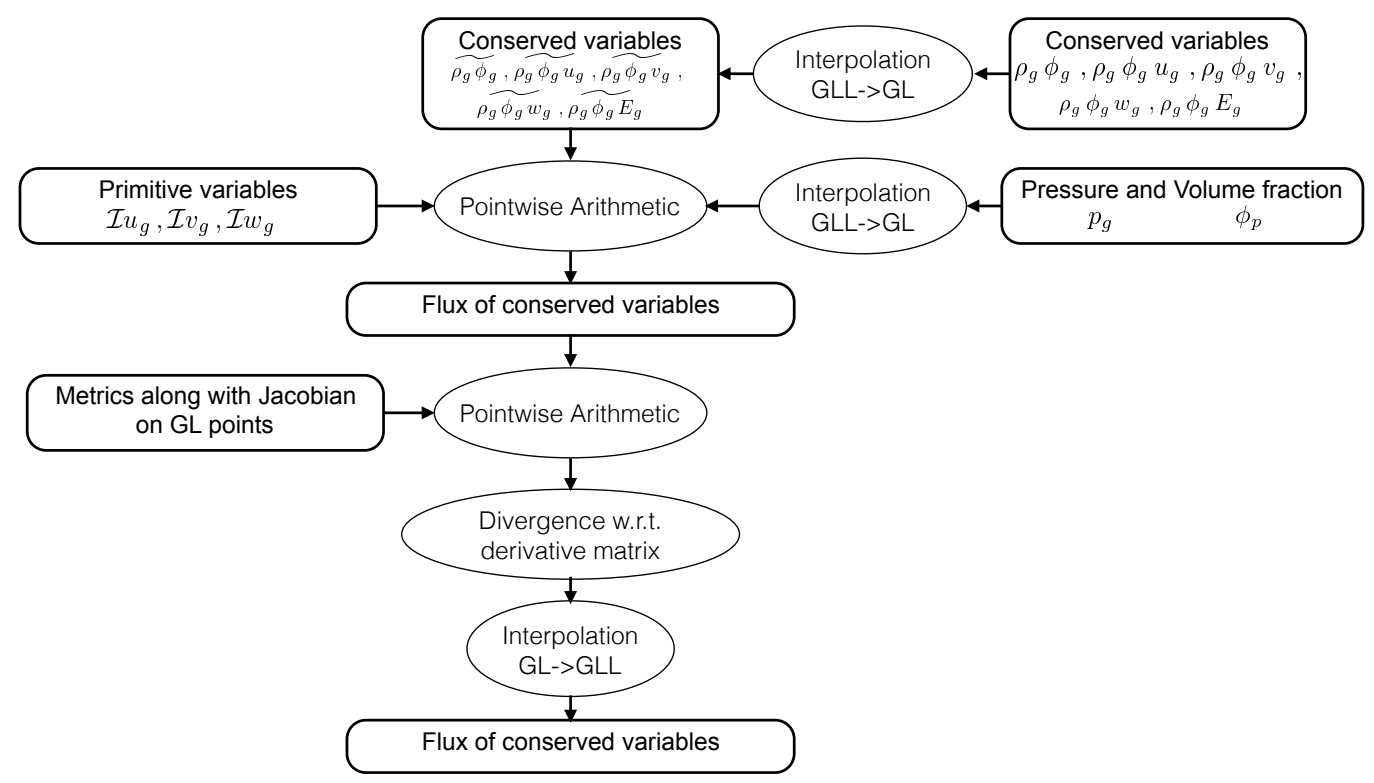

Fig. 5: Workflow of evaluate volume integral step

new location along with updated velocity.

The movement of particles may, in general, require their movement from a spatial domain of a given MPI rank to another MPI rank. This, in general, requires communication between the processes corresponding to the two MPI ranks.

\section{Governing EQuations}

The Eulerian carrier phase is modeled on a fixed mesh by numerical solution of the Euler equations of gas dynamics using a discontinuous Galerkin spectral element method. The dispersed phase is modeled as Lagrangian point particles driven by modeled forces computed from the gas properties interpolated at the particle positions. The artificial viscosity regularization of the Eulerian solver and the back-coupling of unresolved particle forces onto the Eulerian mesh are currently beyond the scope of the CMT-bone proxy application.

The three-dimensional Euler equations of gas dynamics are written in the form of a conservation law

$$
\frac{\partial U_{m}}{\partial t}+\nabla \cdot \mathbf{H}_{m}=R_{m},
$$

where $U_{m}$ is the $m^{\text {th }}$ of five conserved variables,

$$
\mathbf{U}=\phi_{g}\left[\begin{array}{l}
\rho_{g} \\
\rho_{g} u_{g} \\
\rho_{g} v_{g} \\
\rho_{g} w_{g} \\
\rho_{g} E_{g}
\end{array}\right],
$$

for a gas occupying the fraction $\phi_{g}$ of an infinitesimal volume $V$ surrounding a given point $\mathbf{x}$,

$$
\phi_{g}(\mathbf{x}) \equiv \lim _{V \rightarrow 0} \frac{V_{g}}{V} .
$$

The corresponding volume fraction occupied by particles at a given point is $\phi_{p}(\mathbf{x}) \equiv 1-\phi_{g}(\mathbf{x})$. The gas velocity $\mathbf{u}$ and spatial coordinate $\mathrm{x}$ are

$$
\mathbf{u}=\left[\begin{array}{l}
u_{g} \\
v_{g} \\
w_{g}
\end{array}\right]=\left[\begin{array}{l}
u_{1} \\
u_{2} \\
u_{3}
\end{array}\right], \quad \mathbf{x}=\left[\begin{array}{l}
x \\
y \\
z
\end{array}\right]=\left[\begin{array}{l}
x_{1} \\
x_{2} \\
x_{3}
\end{array}\right],
$$

$\rho_{g}$ is the gas density, $E_{g}$ is the mass-specific total energy $e_{g}+\frac{1}{2}|\mathbf{u}|^{2}$ of the gas, $e$ is the gas internal energy, and $p_{g}$ is the thermodynamic gas pressure.

$\mathbf{H}_{m} \in \mathbb{R}^{3}$ is the flux vector of equation $m$. For fluid mass $U_{1}$,

$$
\mathbf{H}_{1}=\phi_{g} \rho_{g} \mathbf{u}=U_{2-4},
$$

and, for gas momentum $U_{2-4}$,

$$
\begin{array}{r}
\mathbf{H}_{2}=\phi_{g}\left[\begin{array}{l}
\left(\rho_{g} u_{g}\right) u_{g}+p_{g} \\
\left(\rho_{g} u_{g}\right) v_{g} \\
\left(\rho_{g} u_{g}\right) w_{g}
\end{array}\right], \mathbf{H}_{3}=\phi_{g}\left[\begin{array}{l}
\left(\rho_{g} v_{g}\right) u_{g} \\
\left(\rho_{g} v_{g}\right) v_{g}+p_{g} \\
\left(\rho_{g} v_{g}\right) w_{g}
\end{array}\right], \\
\mathbf{H}_{4}=\phi_{g}\left[\begin{array}{l}
\left(\rho_{g} w_{g}\right) u_{g} \\
\left(\rho_{g} w_{g}\right) v_{g} \\
\left(\rho_{g} w_{g}\right) w_{g}+p_{g}
\end{array}\right],
\end{array}
$$

and, for gas total energy $\rho_{g} E_{g}$,

$$
\mathbf{H}_{5}=\phi_{g} \mathbf{u}\left(\rho_{g} E_{g}+p_{g}\right) .
$$

The system is closed by an equation of state,

$$
\left[p_{g}, T_{g}\right]=\operatorname{EOS}\left(\rho_{g}, e_{g}\right) .
$$

Treatment of the particles and the source term due to the particles is deferred for the present time. For the present discussion, $R_{m}=0, m=\{1, \ldots, 5\}$ Internal energy per unit 


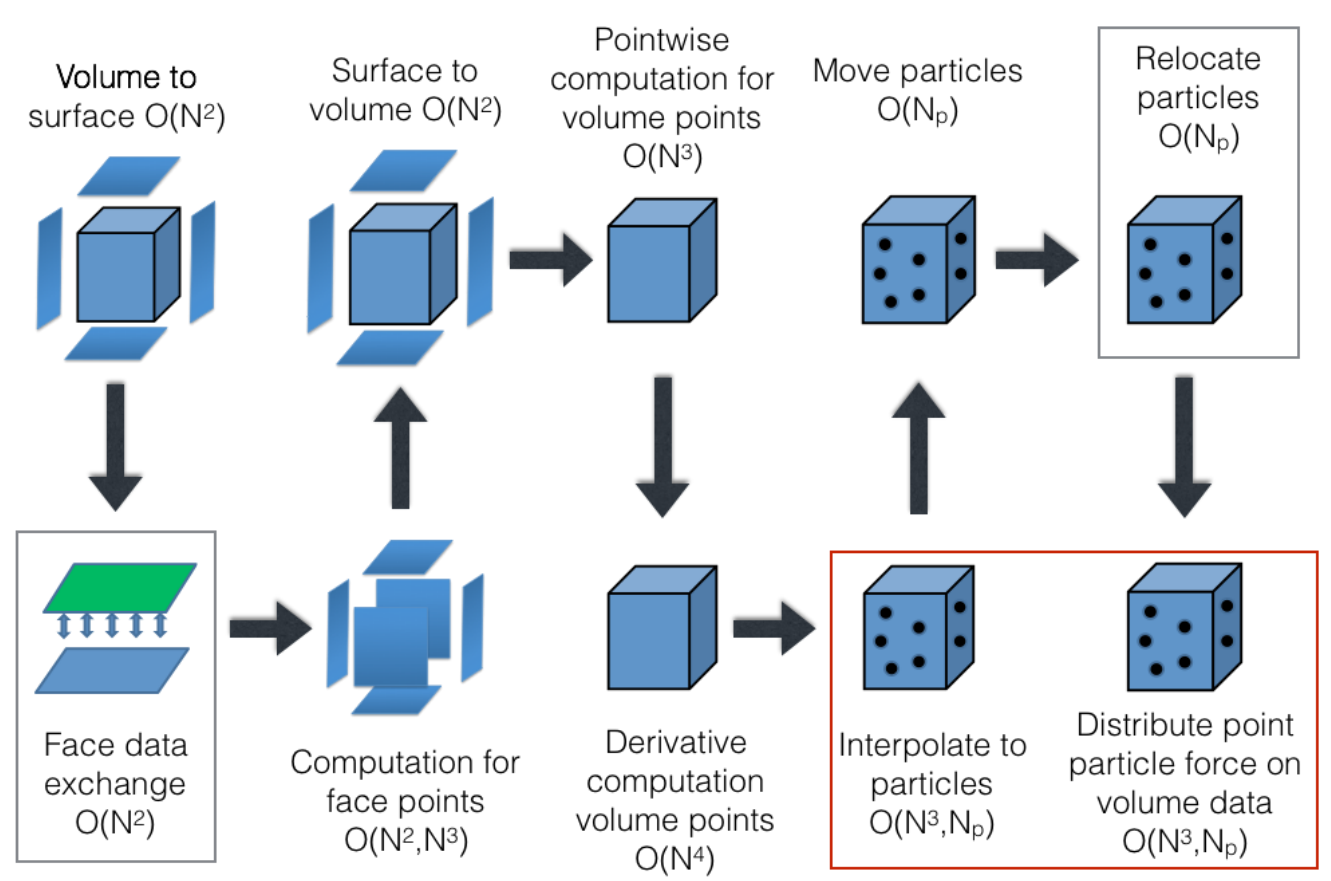

Fig. 6: CMT-nek and CMT-bone workflow that shows the sequence of key compute and communication operations on various data structures

mass $e=E-\frac{1}{2}|\mathbf{u}|^{2}$ is related to gas temperature $T$ by the intensive property $c_{v}$, the constant-volume specific heat, such that

$$
e_{g}=\int c_{v g}(T) d T \text {. }
$$

Generally, Equation 9 must be solved for temperature $T$ implicitly, iteratively, or via tabulation. For calorically perfect gases, $c_{v g}$ is constant. For both thermally and calorically perfect gases, pressure is obtained last via

$$
p_{g}=\rho_{g} R_{g} T_{g},
$$

where the specific gas constant $R=(\gamma-1) c_{v g}$ requires the specification of $\gamma=c_{p g} / c_{v g}$, the ratio of constant-pressure specific heat $c_{p g}$ to $c_{v g}$.

The current version of CMT-nek has one-way coupled point particle tracking capability which means that the point particles are advected based on the forces exerted by the gas, however, the particle motion itself does not modify or affect the surrounding flow. The equation of motion are as shown below

$$
\begin{aligned}
\frac{d \mathbf{x}^{(p)}}{d t} & =\mathbf{v}^{(p)}, \\
\frac{d \mathbf{v}^{(p)}}{d t} & =\frac{1}{m^{(p)}}\left(\mathbf{F}_{Q S}^{(p)}+\mathbf{F}_{S G}^{(p)}+\mathbf{F}_{I U}^{(p)}+\mathbf{F}_{V U}^{(p)}\right) .
\end{aligned}
$$

In the above, superscript $(p)$ refers to the $p^{t h}$ of $M_{p}$ particles. $\mathbf{x}^{(p)}$ and $\mathbf{v}^{(p)}$ is the location and velocity of the point particle $p$. The Eulerian quantities are represented by superscript $(g)$ and refer to interpolated values at particle position $\tilde{x}^{(p)} . \mathbf{F}_{Q S}^{(p)}$ is the quasi-steady drag term, $\mathbf{F}_{S G}^{(p)}$ is the stress gradient force, $\mathbf{F}_{I V}^{(p)}$ is the inviscid-unsteady force and $\mathbf{F}_{V U}^{(p)}$ is the viscous-unsteady force. The expression for these forces are given below

$$
\begin{aligned}
\mathbf{F}_{Q S}^{(p)}= & 3 \pi \mu^{(g)} d^{(p)}\left(\mathbf{u}^{(g)}-\mathbf{v}^{(p)}\right) \\
& f_{1}\left(R e^{(p)}, M^{(p)}\right) f_{2}\left(\phi^{(p)}\right), \\
\mathbf{F}_{S G}^{(p)}= & V^{(p)} \rho^{(g)} \frac{D \mathbf{u}^{(g)}}{D t}, \\
\mathbf{F}_{I U}^{(p)} \approx & \frac{1}{2} V^{(p)} f_{3}\left(M^{(p)}\right) f_{4}\left(\phi^{(p)}\right) \\
& {\left[\rho^{(g)} \frac{D \mathbf{u}^{(g)}}{D t}-\frac{d \rho^{(g)} \mathbf{v}^{(p)}}{d t}\right], } \\
\mathbf{F}_{V U}^{(p)}= & 3 \pi \mu^{(g)} d^{(p)} \int_{-\infty}^{t} K_{V U}\left((t-\xi), \mathbf{R e}^{(p)}\right) \\
& {\left[\rho^{(g)} \frac{D \mathbf{u}^{(g)}}{D t}-\frac{d \rho^{(g)} \mathbf{v}^{(p)}}{d t}\right] d \xi, }
\end{aligned}
$$

where $\mu^{(g)}$ is the viscosity of gas, $\rho^{(g)}$ is the density of gas, $d^{(p)}$ is the diameter of the point particle, $V^{(p)}$ is the volume associated with the point particle, $R e^{(p)}$ is the Reynolds number based on the magnitude of relative velocity of the particle with respective to the surrounding flow, $M a^{(p)}$ is the Mach number based on the relative velocity of the particle. $f_{1}\left(R e^{(p)}, M^{(p)}\right)$ is the finite Mach number and finite Reynolds number correction and $f_{2}\left(\phi^{(p)}\right)$ is the volume fraction correction to the quasi-steady drag term. $f_{3}\left(M^{(p)}\right)$ and $f_{4}\left(\phi^{(p)}\right)$ are 
the finite mach number and volume fraction correction to the inviscid-unsteady (kernel) force term, respectively. is the volume fraction correction to the inviscid-unsteady (kernel) force term. $K_{V U}$ is the viscous-unsteady kernel for computing the viscous unsteady force. Full expressions for these correction terms can be found in [6].

\section{A. Discontinuous Galerkin scheme}

a) The weighted residual statement: Discontinuous Galerkin methods solve Equation 1 by partitioning the domain $\Omega$ into nelt nonoverlapping elements, the $e^{\text {th }}$ of which is $\Omega_{e}$. In the following section, we consider a single conserved variable $U_{m}$ in Equation 2 and omit the subscript $m$. We approximate $U$ by a truncated series of basis functions whose domain is $\Omega_{e}$ and enforcing a weighted-residual statement such that

$$
\int_{\Omega_{e}} v(\mathbf{x}) \frac{\partial U(\mathbf{x})}{\partial t} d V+\int_{\Omega_{e}}(\nabla \cdot \mathbf{H}) v(\mathbf{x}) d V=0
$$

for all test functions $v: \mathbb{R}^{3} \rightarrow \mathbb{R}$ in the same function space where $U$ is approximated[7], [8]. We then integrate the flux divergence $v \nabla \cdot \mathbf{H}$ by parts:

$\int_{\Omega_{e}} v(\mathbf{x}) \frac{\partial U(\mathbf{x})}{\partial t} d V=\int_{\Omega_{e}}(\nabla v) \cdot \mathbf{H} d V-\int_{\partial \Omega_{e}} v(\mathbf{x}) \mathbf{H} \cdot \hat{\mathbf{n}} d A$

where $\hat{\mathbf{n}}(\mathbf{x})$ is the unit normal vector to $\partial \Omega_{e}$ facing outward from $\Omega_{e}$.

The central and distinguishing feature of discontinuous Galerkin methods is the replacement of $\mathbf{H} \cdot \hat{\mathbf{n}}$ in the surface integral in Equation 18 with a numerical flux $\mathbf{H}^{*} \cdot \hat{\mathbf{n}}$ that

1) weakly enforces flux continuity (or boundary conditions should $\partial \Omega_{e} \subset \partial \Omega$ ) at the element interfaces without demanding continuity of $U$ there, and

2) ensures physical correctness of the solution independent of functional representations within the element.

It is important to introduce some notation now; at a given point $\mathbf{x}$ on $\partial \Omega_{e}$,

$$
\begin{aligned}
& U^{-}(\mathbf{x}) \equiv U(\mathbf{x}) \text { taken from the interior of } \Omega_{e} \\
& U^{+}(\mathbf{x}) \equiv U(\mathbf{x}) \text { taken from outside of } \Omega_{e}
\end{aligned}
$$

where $\mathbf{U}^{+}$is understood to be equal to $\mathbf{U}^{-}$for the neighboring element sharing $\partial \Omega_{e}$ with the $e^{\text {th }}$ element. The numerical flux must be consistent:

$$
\mathbf{H}^{*}\left(\mathbf{U}^{-}, \mathbf{U}^{+}\right)=\mathbf{H}(\mathbf{U}) \text { if } \mathbf{U}^{+}=\mathbf{U}^{-},
$$

and will be discussed further in later sections.

b) Spectral element discretization: Inside the $e^{\text {th }}$ element $\Omega_{e}$ the unknowns $\mathbf{U}$ are represented in some finite-dimensional space of functions $\chi$. The representation of a single (scalar component of Equation 2) conserved variable $U$ is written as a truncated series expansion

$$
U(x) \approx \sum_{i=1}^{N} \widehat{U}_{i} \phi_{i}(x), x \in \Omega_{e}
$$

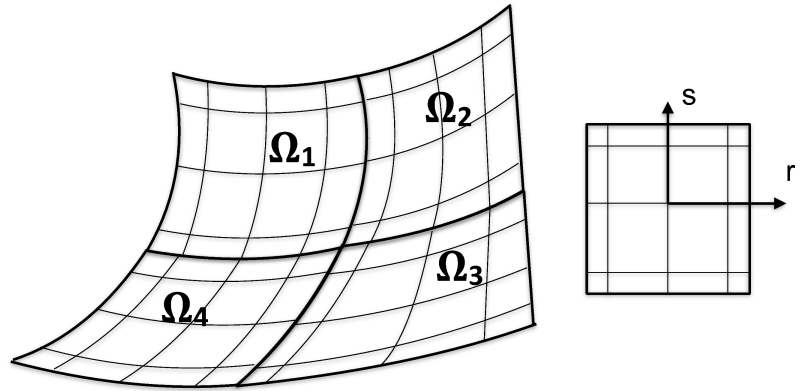

Fig. 7: A schematic representation of a collection of spectral elements making up a computational domain. A schematic representation of a reference element is also shown.

that is, a linear combination of basis functions spanning $\chi$ $\left\{\phi_{i}: \Omega_{e} \rightarrow \mathbb{R}, i=1, \ldots N\right\} \subset \chi$, each of which is weighted by some coefficient $\widehat{U}_{i}$. Note that Equation (22) refers to a one-dimensional problem. In this simplification the element is just a line segment. Furthermore,

1) Coordinate $x$ in each element is isoparametrically mapped onto the reference element $r \in[-1,1]$. Figure 7 shows a schematic of 2 dimensional space divided into elements. Also a schematic of a reference element is shown in the figure.

- This transformation has, $\forall \mathbf{x} \in \Omega_{e}$ :

a) $\operatorname{Jacobian} \operatorname{Jac}(\mathbf{x}) \equiv|\partial \mathbf{x} / \partial \mathbf{r}|$

b) Metrics $\partial \mathbf{r} / \partial \mathbf{x}$

- $\chi$ is the space of $(N-1)^{\text {th }}$-order polynomials $\mathbb{P}^{N-1}$.

- The coefficients $\widehat{U}_{i}=U\left(x_{i}\right), i=1 \ldots N$ are the values of the unknown at grid points $x_{i}$ within $\Omega_{e}$. Thus, Equation 22 is said to be a nodal approximation to $U$.

- The $i^{\text {th }}$ basis function $\phi_{i}=l_{i}(x)$ is the Lagrange interpolating polynomial associated with the $i^{\text {th }}$ node $x_{i}$.

- This representation extends to higher dimensions by tensor-nesting the Lagrange polynomials in each of the coordinate directions. For example, in three dimensional space we have directions $r, s$, and $t$ on the cubic $[-1,1]^{3}$ reference element.

2) The grid points on the reference element are the $N$ Gauss-Legendre-Lobatto (GLL) quadrature nodes[9] .

The next major numerical approximation underlying the method is to approximate all integrals by Gaussian quadrature on the $N$ GLL points in the reference element. Quadrature approximates an integral by a weighted sum of nodal values,

$$
\int_{-1}^{1} f(r) d r \approx \sum_{i=1}^{N} \omega_{i} f\left(r_{i}\right)
$$

where the quadrature weight for the $i^{\text {th }}$ point $\omega_{i}$ depends on the locations of the quadrature nodes $r_{i}$. Formulas for determining quadrature weights for the GLL nodes are wellknown [7], [9]. 
Nodal values at grid points are arranged into vectors along a given reference-element coordinate ( $r$ is shown):

$\mathbf{U} \equiv\left[\begin{array}{c}U\left(r_{1}\right) \\ \vdots \\ U\left(r_{N}\right)\end{array}\right], \mathbf{v} \equiv\left[\begin{array}{c}v\left(r_{1}\right) \\ \vdots \\ v\left(r_{N}\right)\end{array}\right], \mathbf{h}_{1} \equiv\left[\begin{array}{c}H_{x}\left(U\left(r_{1}\right)\right) \\ \vdots \\ H_{x}\left(U\left(r_{N}\right)\right)\end{array}\right]$

Then defining

$$
\operatorname{diag}(\mathbf{v}) \equiv\left[\begin{array}{ccc}
\searrow & & 0 \\
& v\left(r_{i}\right) & \\
0 & & \searrow
\end{array}\right]
$$

will allow us to write vectors of $N$ nodal values as $N \times N$ diagonal matrices:

$$
\begin{aligned}
J & \equiv \operatorname{diag}(\mathrm{Jac}) \\
\mathcal{M} & \equiv \operatorname{diag}\left(\omega_{j}\right) .
\end{aligned}
$$

Finally, the derivative of a function approximated by Equation 22 at its $N$ nodes $r_{i}$ may itself be approximated by finite differences. Many authors[7], [8], [10] provide the details of how to represent $(N-1)^{\text {th }}$-order-accurate finite differences on each of $N$ points as matrix-vector products between a $N \times N$ differentiation matrix $\mathcal{D}$ and a vector of nodal values:

$$
\mathcal{D} \mathbf{v} \approx\left[\left.\frac{d v}{d r}\right|_{r_{1}}, \cdots,\left.\frac{d v}{d r}\right|_{r_{N}}\right]^{\top}
$$

For the rest of this section, subscripts refer to Cartesian components of vectors in $\mathbb{R}^{3}$, so $r_{j}$ is the $j^{\text {th }}$ component of $\left(r_{1}, r_{2}, r_{3}\right)^{\top}=(r, s, t)^{\top}$ and $x_{i}$ is the $i^{\text {th }}$ component of $\mathbf{x}$ (Equation 4$). \mathcal{D}_{j}$ refers to an appropriately tensor-nested differentiation operator in the direction of $r_{j}$ ([7], Chapter 4). Einstein summation convention is applied to spatial coordinates, and bold-faced quantities are vectors of grid points (Equation 24), not spatial vectors. $\mathcal{M}$ is also tensor-nested: $M_{m n o}=\omega_{m} \omega_{n} \omega_{o}$ for the grid point with indices $m, n, o$.

Additionally defining $\mathcal{E}$ as an indicator that is zero for all grid points except those on the element faces $\partial \Omega_{e}$, the discontinuous Galerkin weighted-residual statement Equation 18 may be written as a semidiscrete governing equation in matrix form as

$$
\begin{aligned}
\mathbf{v}^{\top}\left[J \mathcal{M} \frac{\partial \mathbf{u}}{\partial t}\right] \approx\left[\left[\operatorname{diag}\left(\frac{d r_{i}}{d x_{j}}\right)\right] \mathcal{D}_{i} \mathbf{v}\right]^{\top} & {\left[\mathcal{M} \mathbf{h}_{j}\right] } \\
& -\mathbf{v}^{\top}\left[\mathcal{E} \mathcal{A}\left(J_{A} \mathbf{h}_{j}^{*} \hat{n}_{j}\right)\right.
\end{aligned}
$$

where $J_{A}$ is the Jacobian of the transformation mapping a given face (selected by $\mathcal{E}$ ) to the reference $[-1,1]^{2}$ square. By equating coefficients of the nodal values of the test function $\mathbf{v}$, the weighted residual theorem is satisfied for all possible $v \in \chi$ and the semidiscrete system reads (for the vector of $N$ GLL points in a given element along a given line in $r s t$ space)

$$
\mathcal{M} \frac{\partial \mathbf{u}}{\partial t}=\mathcal{D}_{i}^{\top}\left[\operatorname{diag}\left(\frac{1}{J} \frac{d r_{i}}{d x_{j}}\right) \mathcal{M} \mathbf{h}_{j}\right]-\mathcal{E}\left[\mathcal{A}\left(\frac{J_{A}}{J} \mathbf{h}_{j}^{*} \hat{n}_{j}\right)\right],
$$

where parentheses represent scalar collocation of different values with one another at the same grid point.

c) Managing aliasing error: It is well-known that Gaussian quadrature on $N$ GLL points exactly integrates a polynomial of order $2(N-1)-1$. However, the test function, unknowns, and field variables like pressure and velocity are all polynomials of order $N-1$; their products produce integrands that may not be exactly integrated on only $N$ points. The functional forms of the Jacobian and the metrics also affect how many points are needed for exact quadrature, especially on deformed elements with curved faces. Errors from inexact quadrature are said to "alias" onto the degrees of freedom, and the provision of more grid points for quadrature is called "overintegration[11]" or dealiasing.

The standard sources [7], [8], [10] on high-order methods based on polynomials also represent polynomial interpolation from $N$ points represented by Equation 22 to $M$ other points as a product between an $M \times N$ interpolation matrix $\mathcal{I}$ and $N$-vectors like those in Equation 24. Avoiding the details of such an operation, let

$$
\mathcal{I} \mathbf{v}=\left[\begin{array}{c}
v\left(r_{1}\right) \\
\vdots \\
v\left(r_{M}\right)
\end{array}\right]
$$

be the matrix-vector product that interpolates $\mathbf{v}$ from $N$ GLL points to $M>N$ Gauss-Legendre (GL) quadrature nodes, a slightly different choice of nodes with quadrature weights that can integrate polynomials of order $2 M-1$ exactly. Considering a longitudinal flux of momentum (e.g., $z$-momentum in the $z$ direction), Equation 6 says

$$
H_{z}=(\rho w) w_{g}+p_{g}=U_{4} w_{g}+p_{g}
$$

An inner product of the interpolating polynomial Equation 22 representing $H_{z}$ in Equation 32 with that of the test function gradient $\partial v / \partial r_{j}$ would be the integral of the product of three polynomials: $U_{4} \& w$ (both $^{2} \in \mathbb{P}^{N-1}$ ), and $\partial v / \partial r_{j} \in \mathbb{P}^{N-2}$. This integrand has degree $3 N-4$, and to avoid multiplicative aliasing errors, Gaussian quadrature (Equation 23) needs each polynomial in Equation 32 on $M$ GL points such that $2 M-1>$ $3 N-4$. Thus, $M=3(N-1) / 2$ (or greater, depending on the polynomial order of the grid metrics and Jacobian), and the vector of grid points $\mathbf{h}_{3}$ must be formed by interpolating $\mathbf{U}_{4}$, w and $\mathbf{p}$ from the $N$-point GLL grid to the $M$-point GL grid, forming

$$
\mathbf{h}_{3, M}=\left[\operatorname{diag}\left(\mathcal{I} \mathbf{U}_{4}\right)\right]\left[\mathcal{I} \mathbf{w}_{g}\right]+\mathcal{I} \mathbf{p}_{g}
$$

interpolating the metrics onto the $M$-point grid, and repeating the manipulations that led from Equation 29 to Equation 30. The first right-hand-side term in Equation 30

$$
\mathcal{D}_{i}^{\top}\left[\operatorname{diag}\left(\frac{1}{J} \frac{d r_{i}}{d x_{j}}\right) \mathcal{M} \mathbf{h}_{j}\right],
$$

\footnotetext{
${ }^{2} w=U_{4} / U_{1}$ is technically a rational function, but it responds favorably to overintegration. We treat the thermodynamic pressure $p$ as a polynomial in spite of the nonlinearity of Equation 8.
} 
is replaced by

$$
\mathcal{I}^{\top} \mathcal{D}_{i, M}^{\top}\left[\operatorname{diag}\left(\mathcal{I}\left[\frac{1}{J} \frac{d r_{i}}{d x_{j}}\right]\right) \mathcal{M}_{M} \mathbf{h}_{j, M}\right],
$$

where the subscript $M$ denotes the both the mass matrix and differentiation operators that have been independently computed for $M$ Gauss-Legendre points.

The surface integral in Equation 30 must be re-evaluated in the same way. The interpolation operator $\mathcal{I}$ is tensornested; it is multiplied by a vector of point values along each "line" of GLL points for each line in each of three directions successively. The operator $\mathcal{I}_{A}$ is the 1D GLL interpolation matrix nested twice instead of three times, and it interpolates grid points on an element face from a 2D plane of GLL points to a $2 \mathrm{D}$ plane of Gauss-Legendre points. Likewise, we use a diagonal matrix $\mathcal{A}$, whose elements are $M_{i j}=\omega_{i} \omega_{j}$ at a given crossing of GLL lines, to handle quadrature for surface integrals.

$$
\mathcal{E A}\left(\frac{J_{A}}{J} \mathbf{h}_{j}^{*} \hat{n}_{j}\right)
$$

becomes

$$
\mathcal{E} \mathcal{I}_{A}^{\top}\left[\mathcal{A}_{M} \operatorname{diag}\left(\mathcal{I}_{A} \frac{J_{A}}{J}\right) \operatorname{diag}\left(\mathbf{h}_{j, M}^{*}\right)\left[\mathcal{I}_{A} \hat{\mathbf{n}}_{j}\right]\right],
$$

where both the surface normal vector $n_{j}$ and the Jacobian ratio $J_{A} / J$ have been interpolated onto the $M^{2}$ GL face points.

The only remaining steps are to substitute Equations 35 and 37 into Equation 30 and multiply through by $\mathcal{M}^{-1}$ to get our final semi-discrete system.

$$
\begin{aligned}
\frac{\partial \mathbf{u}}{\partial t} & =\mathcal{M}^{-1} \mathcal{I}^{\top} \mathcal{D}_{i, M}^{\top}\left[\operatorname{diag}\left(\mathcal{I}\left[\frac{1}{J} \frac{d r_{i}}{d x_{j}}\right]\right) \mathcal{M}_{M} \mathbf{h}_{j, M}\right] \\
& -\mathcal{M}^{-1} \mathcal{E I}_{A}^{\top}\left[\mathcal{A}_{M} \operatorname{diag}\left(\mathcal{I}_{A} \frac{J_{A}}{J}\right) \operatorname{diag}\left(\mathbf{h}_{j, M}^{*}\right)\left[\mathcal{I}_{A} \hat{\mathbf{n}}_{j}\right]\right],
\end{aligned}
$$

We note here that, ordinarily, inverting the mass matrix "lifts" surface integrals into volume nodes. Under the assumptions of spectral element methods, however, the mass matrix is diagonal by construction, and no lifting occurs; surface integrals only directly affect surface nodes.

\section{CMT-BOnE}

CMT-nek is being developed by the researchers at University of Florida and other institutions for performing large scale simulation of compressible multiphase turbulence on large scale supercomputers. The goal is to achieve the highest available performance while minimizing power or energy requirements to best utilize state of the art supercomputers at a variety of lab computing facilities. This will require a variety of algorithmic, software and numeric changes to the code. Direct use of the parent application to evaluate the impact of these choices can be impractical and cumbersome. The use of a representative proxy app provides a simpler framework for software developers and application scientists to investigate the multitude of hardware and software choices. A small subset of these choices can then be transferred to the parent application to achieve close to the best achievable performance.

Achieving a representative proxy application requires that the key data structures and corresponding computational and communication structures from the parent application are present. Figure ?? shows an abstract representation that highlights the important arrays, computations and communication operations in CMT-nek. To put this schematic in the context of the implementation details and the workflows, we can clearly see the following

- The surface integral evaluation described in II-B is represented by the first four blocks in Figure ??. A close inspection of Figure 4 and Figure ?? shows that the "full_to_face" step is represented by the "Volume to surface" block, and "GS_op" step is represented by the "Face data exchange" block. The "computation for face points" is an abstraction of the "AUSM+" and "Boundary condition" step in Figure 4. Finally, the "face_to_full" step in Figure 4 is represented by the "Surface to volume" block in Figure ??.

- The next two blocks - "Pointwise computation for volume points", and "Derivative computation volume points" represent the evaluate volume integral section described in II-C.

- The last four blocks represent the Lagrangian point particle tracking in CMT-nek. Note that the last block, namely, "Distribute point particle force on volume data", is currently under development and therefore not described in the earlier sections.

CMT-bone has all of the above steps and fully retains the workflow of CMT-nek. The main simplification is the number of allocated variables and corresponding fewer computation and communication steps that are performed. CMT-bone defines variables for the five conserved variables of CMT-nek (mass, energy, and the three components of momentum). It also uses a subset of primitive variables used in CMT-nek such as pressure, density and velocity. Particle processing in CMT-bone is similar to that in CMT-nek.

CMT-bone processes operations described in Sections II-A, II-B, II-C. but simplifies computations corresponding to computation of diffusive fluxes and treatment of viscous flows. Our goal is to evolve CMT-bone along with the most mature version of CMT-nek so that it inherits all of the key abstractions, computations and communication structures.

Table II provides a list of variables used in CMT-bone and corresponding variables used in CMT-nek. We also provide a comparative cardinality of the actual number of variables used. The corresponding workflow is shown in Figure ??.

\section{VALIDATION APPROACH}

CMT-bone and CMT-nek were compared in terms of performance to identify the representative utilization of resources. All experiments were run on a 431 TFlop Intel Xeon Sandy Bridge cluster, $\mathrm{Cab}$, located at the Lawrence Livermore National Laboratory. The cluster consists of 1,296 nodes, where each node is a dual socket with 8 cores on each socket. Each 
TABLE II: Key subroutines in CMT-nek implementation and their corresponding description in the CMT-nek workflow shown in Figure ??.

\begin{tabular}{ccccc}
\hline step & CMT-nek subroutine & CMT-nek workflow description & \multicolumn{2}{c}{ number of variables } \\
& & & CMT-nek & CMT-bone \\
\hline 1 & primitive_variables & pointwise computation for volume points & 9 & 5 \\
2 & fillq & Volume to surface & 15 & 10 \\
3 & gs_op & face data exchange & 18 & 10 \\
4 & ausm & computation for face points & 5 & 5 \\
5 & addfacetofull & surface to volume & 5 & 5 \\
6 & evaluate_conv_h & Pointwise compuation for volume points & 15 & 15 \\
7 & flux_div_integral & Derivative compuation for volume points & 15 & 15 \\
8 & baryweights_findpts_eval & Interpolate to particles & 3 & 3 \\
9 & update_stokes_particles & Move particles & 3 & 3 \\
10 & crystal_tuple_transfer & Relocate particles & 3 & 3 \\
11 & & Distribute point particle force on volume data & - & - \\
\hline
\end{tabular}

node has 32 GB memory. The cache subsystem consists of private $\mathrm{L} 1$ data and instruction caches of size $32 \mathrm{~KB}$, private L2 cache of size $256 \mathrm{~KB}$ and a shared L3 cache of size 20 MB. Total memory bandwidth is $51.2 \mathrm{~GB} / \mathrm{s}$.

\section{A. Input Parameters}

The four important pieces of the CMT-nek flow, executed in each time step, may be described as:

1) Point computations: point_compute_kernel computes the primitive variables for each grid point.

2) Communication step: comm_kernel encapsulates the communication code that is used to share surface data among neighbors.

3) Computation step: compute_kernel contains the key computations involving the fluxes and their partial spatial derivatives.

4) Processing particles: particles_kernel initializes, distributes and tracks particles.

The four kernels above are also present in a reduced form in CMT-bone, except for the particles_kernel. Since particles_kernel is under development at the time of writing this paper, it is bare bones and hence replicated as such in CMT-bone. Consequentially, we did not validate the particles_kernel.

Performance validation was done for the three remaining kernels on a single node by observing performance characteristics as CMT-nek and CMT-bone were scaled from 1 through 16 processes on a node. The workload was changed by considering different polynomial orders as $5,7,9,11,13,15^{3}$ and different total number of spectral elements as 512 and 1024.

\section{B. Resource Groups}

Islam et al. in [3] developed VERITAS, a validation framework for proxy applications. In this paper we use VERITAS for on-node comparison of performance of CMT-nek and CMTbone. The verification is based on values recorded by the hardware performance counters. The performance counters available on an architecture are categorized into resource

\footnotetext{
${ }^{3}$ Odd $N_{x}$ ensures even polynomial order and integral whole numbers for $M$, the number of points for overintegration.
}

TABLE III: Resource groups for Intel Xeon

\begin{tabular}{l|l} 
Resource Group & Intel Xeon \\
\hline Floating point unit & FP \\
Branch unit & BR \\
Prefetch events & PREFETCH \\
L1 cache & L1 \\
L2 cache & L2 \\
Last level L3 cache & L3 \\
Translation look aside buffer & TLB \\
Memory & MEM \\
Remote socket traffic & OFFCORE \\
PCI express bus & PCIE
\end{tabular}

TABLE IV: Workloads used in our experiments

\begin{tabular}{c|c|c} 
Number of Elements & Workload Id & Polynomial Order \\
\hline \multirow{3}{*}{512} & 1 & 5 \\
& 2 & 7 \\
& 3 & 9 \\
4 & 4 & 11 \\
& 5 & 13 \\
& 6 & 15 \\
\hline \multirow{5}{*}{1024} & 7 & 5 \\
& 8 & 7 \\
& 9 & 9 \\
& 10 & 11 \\
& 11 & 13 \\
\hline
\end{tabular}

groups. Machine learning techniques are then used to relate the utilization of the resources on an architecture by both parent and proxy applications to the efficiency loss as the application is scaled. The high-level resource groups on an Intel Xeon platform are presented in Table III.

\section{Validation Methodology}

The validation methodology of VERITAS is explained as follows. The two primary steps in the methodology are (1) detecting the resource groups that present themselves as bottlenecks for scalability of the parent application (CMT-nek) (2) detecting the resource groups that are bottlenecks for scaling the proxy application (CMT-bone).

1) Identifying important resources: Resources important to an application are those that are heavily used in the application and quickly become bottlenecks especially when the application is scaled. A reference used by VERITAS to find important resources is efficiency loss as the application scales. It is normal to observe an increase in efficiency loss as more cores are used on a multi-core machine to run an application. Because all performance events have a certain 
penalty, VERITAS calculates the total penalty for all performance metrics to contrast with efficiency loss of the original application. In fact, the more precise the performance metric penalty is in relation to the the growth of the efficiency loss as a application scales, the more important the metric and the corresponding resource become. VERITAS is configured with the performance metrics gathered from CMT-nek workloads and recognizes a sparse subset of performance metrics which highlight characteristics of efficiency loss and uses the metrics to construct a predictive linear model. Utilizing the inferred sparse model, VERITAS merges the importance values of all metrics related to a resource to estimate the importance of the resource. Hence, VERITAS equips users with a comprehensive understanding of the correct hardware resources which affect the performance in a proxy application, as well as the actual importance of these resources in establishing the performance of the parent application. This awareness allows users to develop a proxy application that effectively covers the hardware resources which are essential to deducing the behavior of the parent application. In a sparse model the underlying theory is that the data points are drawn from a union of low dimensional subspaces that might not be completely disjoint or orthogonal. VERITAS utilizes the idea that a compound pattern can be effectively decomposed into a small set of simple elementary patterns. Mathematically, this assumption is expressed as follows. Given a data sample $\mathbf{y} \in \mathbb{R}^{n}$ and a dictionary of $K$ representative patterns $\mathbf{D} \in \mathbb{R}^{n \times K}$, the sparse representation $\mathbf{a} \in \mathbb{R}^{K}$ can be obtained as

$$
\min _{\mathbf{a}}\|\mathbf{y}-\mathbf{D a}\|_{2}^{2} \text { s.t. }\|\mathbf{a}\|_{0} \leq \kappa .
$$

where $\|\cdot\|_{0}$ denotes the $\ell_{0}$ norm that counts the total number of non-zero entries in a vector, \|\|$_{2}$ is the $\ell_{2}$ norm, and $\kappa$ is the desired sparsity. The second constraint imposes nonnegativity on the solution and results in an additive model that is easy to interpret. In our case, $\mathbf{y}$ is the efficiency loss (or loss in runtime), $n$ is the number of workers (or the number of workloads), the dictionary $\mathbf{D}$ is the collection of $K$ performance metrics.

It is noted that this optimization problem solves for the sparsest set of performance metrics that can reconstruct the efficiency loss. In particular, VERITAS uses the Orthogonal Matching Pursuit (OMP) algorithm proposed in [12] to select that smallest diverse subset of metrics to describe the performance loss.

The two parameters usable in VERITAS to fine tune the modeling of efficiency loss are complexity and sparsity. While the complexity parameter determines the number or resources needed in the model to represent the performance characteristics, the sparsity parameter determines the number of counters per resource that are considered. A higher value of complexity indicates more resources will be used in the model to explain performance loss. Thus, the complexity parameter may be used to identify higher-order resources that will become a bottleneck if the immediate bottleneck is removed. On the other hand, a higher value of sparsity increases the number of counters and includes counters that contribute only marginally.
The overall goal is to build a simple model with sparsity high enough that only the dominant counters are selected.

2) Comparing proxy to parent: The main strategy to compare a proxy application to the parent application is to study the feature spaces defined by the performance metrics for the two applications and measuring the commonality between these feature spaces. As it is common in statistical modeling to assume that the underlying data distribution may be represented using fewer degrees of freedom, similarly VERITAS compares the high-dimensional feature spaces using the corresponding low-dimensional representatiions determined using Principal Component Analysis (PCA). A linear subspace can be conveniently represented using its basis $\mathbf{B} \in \mathbb{R}^{C \times d}$, where $C$ is the dimensionality of the data, and $d$ is the dimensionality of the subspace. The collection of all $d$-dimensional linear subspaces forms the Grassmannian $G(d, C)$, a smooth Riemannian manifold, which allows effective geometric and statistical analysis of subspaces. VERITAS constructs the geodesic curve between the two subspaces and estimates a dissimilarity measure between them using the computed dissimilarity, which is an inverse of compatibility. Thus, the proxy and parent applications are considered to be compatible for a resource group when (1) the two sub-spaces are well aligned geometrically, which is determined by computing the angles between basic vectors along the principle components and (2) the data in the projected subspaces are distributed similarly. When multiple workloads are used, the dissimilarity measure is obtained as the average of individual workloads.

VERITAS defines the Coverage measure for each resource $r$ as

$$
\text { Coverage }_{r}=1-\text { dissimilarity }_{r}, \forall r \text {. }
$$

\section{Interpreting Results}

In this section we will give a brief primer about how to interpret the results and charts from VERITAS using Figure 9a. Resource importance in predicting efficiency loss is plotted on the X-axis, while coverage (or quality of match) for each resource is plotted along the Y-axis. Resource importance has a value between 0 and 1 where 0 means the lowest and 1 means the highest importance. Coverage has a value also between 0 and 1 where 0 means "does not cover" and 1 means "covers $100 \%$ of the behavior". For example, Coverage $\geq 0.9$ means that on average the proxy covers more than $90 \%$ of the utilization behavior of a certain resource. Similarly, Resource Importance $\geq 0.7$ means that on average a resource utilization behavior affects scalability of an application with more than $70 \%$ probability. The user may define thresholds for both resource importance and coverage and pass it to VERITAS. In our experiments we specified $80 \%$ as the threshold for both coverage and resource importance, which means Coverage $\geq$ 0.8 is good coverage, and Resource Importance $\geq 0.8$ are important to cover (hence the dotted line through 0.8).

\section{RESULTS}

In this section we first demonstrate how the user may choose the sparsity and the complexity parameters, and then present 


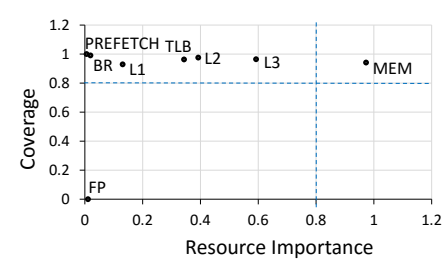

(a) $\kappa=10$

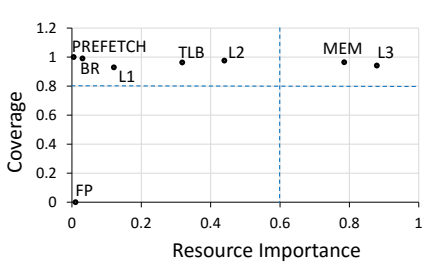

(b) $\kappa=15$
Fig. 8: Results for different $\kappa$ for $\tau=10$

actual validation results from our experiments in Sections VI-B through VI-D.

\section{A. Determining Parameters:}

To identify the best model parameters $\kappa$ (sparsity) and $\tau$ (model complexity) for a given kernel, we vary both of these parameters and determine a reasonable setting that is used for actually analyzing the kernel. We present the results of the compute_kernel in Figure 8 for different values of parameters $\kappa$ and $\tau$. Though other combinations of parameter values were also considered for the compute_kernel as well as the remaining kernels, we present the results only for the chosen combinations here in the interest of space. We observe that decreasing sparsity (increasing $\kappa$, i.e. involving more performance metrics for a resource) does not significantly change the importance of resources. Also, using $\tau \geq 10$ or more encourages OMP to include different resources in the solutions. This phenomenon results in L3 resource becoming more important. Higher values of $\tau$ or $\kappa$ do not change the importance of the resources in any significant way. Hence, for the rest of the experiments with compute_kernel, we used $\kappa=15$ and $\tau=10$.

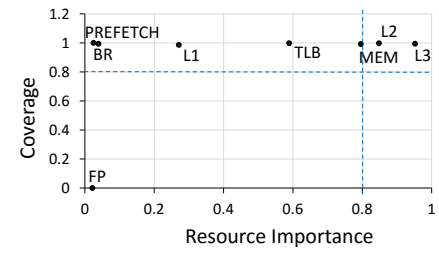

(a) point_compute_kernel

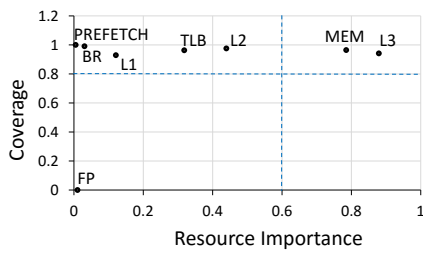

(b) compute_kernel
Fig. 9: CMT-bone captures all the resource utilization behavior of CMT-nek for the two kernels.

\section{B. Point computations:}

The computations performed in the point_compute_kernel are vector-vector multiplications to compute the values of primitive variables at each grid point. A comparison of resource utilization behavior of the kernel for CMT-nek and CMT-bone for kernel validation between proxy and parent application is shown in Figure 9a. The memory utilization behavior of CMT-nek is the most predictive of the efficiency loss of point_compute_kernel with scale in CMT-nek and the corresponding kernel in CMT-bone capturing that behavior very well (with Coverage $>0.8$ ).
Figure 9a also shows that the performance behavior of the point_compute_kernel in CMT-bone matches all resource utilization behavior of the kernel in CMT-nek on Xeon, hence it is an ideal proxy.

\section{Computation step:}

The computations performed in the compute_kernel are matrix-matrix multiplications to compute the values of fluxes and partial derivatives of the fluxes. Figure $9 \mathrm{~b}$ reveals that the major cause for efficiency loss with scale in the compute_kernel of CMT-nek is L3 and node memory utilization and CMT-bone covers these resource behavior perfectly. Further investigation reveals that the gradient and the volume integral computations in this module perform a number of matrix-matrix multiplications where matrices are accessed in strides of $1, N$ and $N^{2}$. This results in a large number of L3 store misses which in turn generates a large number of node memory store operations.

\section{Communication step:}

Even though the performance of this module is dominated by inter-node communication, our analysis identifies the key computation behavior that cause efficiency loss in this kernel with scale, since we collect on-node hardware performance counters.

Figure 10a shows that even though memory utilization is the most important behavior that impacts scalability (Resource Importance $\geq 0.9$ ) of CMT-nek, the comm_kernel in CMT-bone does not capture this behavior (Coverage $<0.5)$.

Figure $10 \mathrm{~b}$ shows the performance characteristics for each workload with scale and it can be seen that the performance behavior of CMT-nek and CMT-bone differs significantly for the different workloads especially for resources such as MEM.

The runtimes for CMT-bone and CMT-nek for the four different regions corresponding to workload 12 are plotted in Figures 11 through 13. These figures show that runtimes for both the applications are comparable.

\section{RELATED WORK}

The CORAL benchmarks [13], developed collaboratively by scientists and engineers at the three national laboratories, namely, Oak Ridge National Laboratory (ORNL), Argonne National Laboratory (ANL) and Lawrence Livermore National Laboratory (LLNL), contain a good collection of benchmarks and proxy applications. We will present some of these applications in this section.

The first application, related to our work, is Nekbone [14], which is a proxy application for Nek5000. The kernels captured by Nekbone are representative of those carrying out the key computation and communication function of Nek5000. Thus, Nekbone solves a standard Poisson equation using the spectral element method with an iterative conjugate gradient solver along with a simple preconditioner. In the spectral element method data decomposition is done by partitioning the entire computational domain into higher-order quadrilateral 


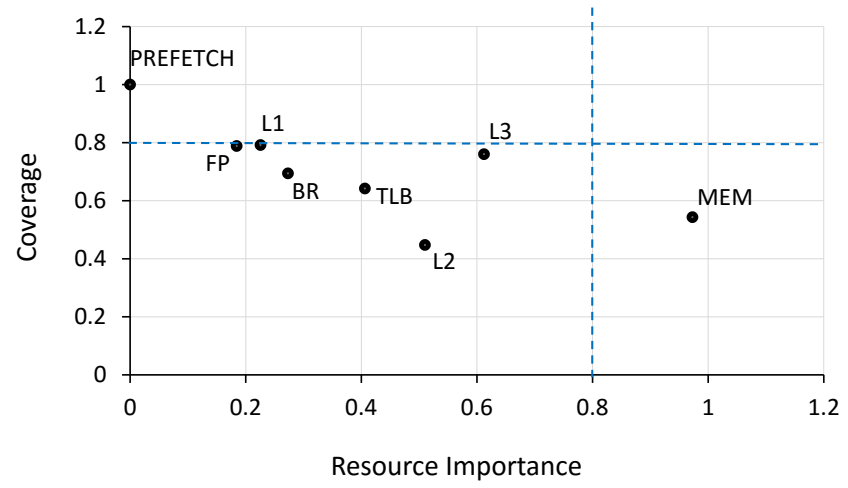

(a) Resource utilization of CMT-nek and coverage of CMT-bone.

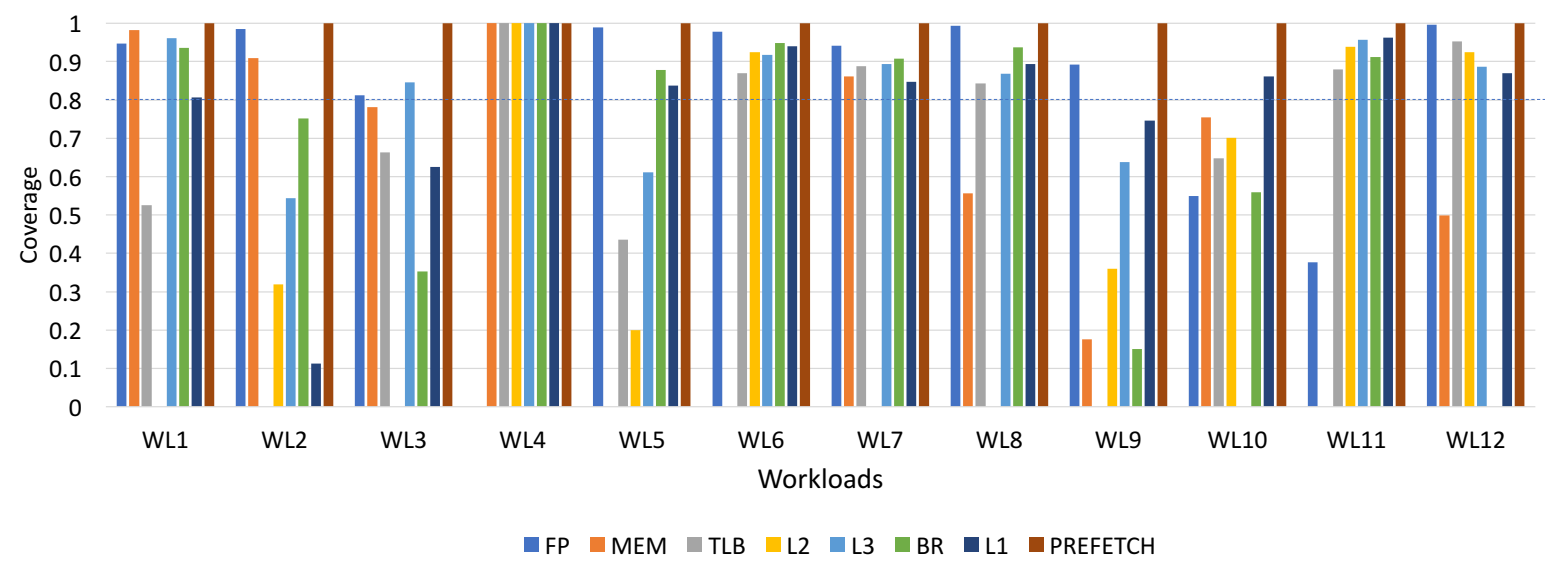

(b) The MEM resource coverage is poor for most of the workloads

Fig. 10: For comm_kernel, CMT-bone does not cover the memory utilization behavior of CMT-nek with scale for multiple workloads.

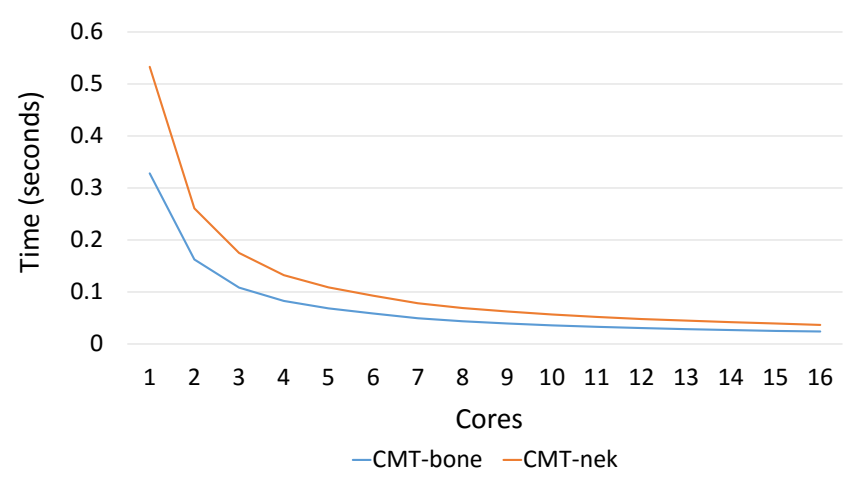

Fig. 11: Comparison of run times of CMT-nek and CMT-bone for the point_compute_kernel.

elements. These elements are then mapped to the processors using a recursive spectral bisection technique.

AMG2013 [15] is a proxy application for the BoomerAMG solver as part of the hypre library at LLNL. AMG2013 [15] is a parallel algebraic multi-grid solver that solves a linear system of equations arising from problems on unstructured grids. The code is synchronous and reveals surface-to-volume relationship in its communication as well as computation patterns. The proxy app is memory intensive having very low

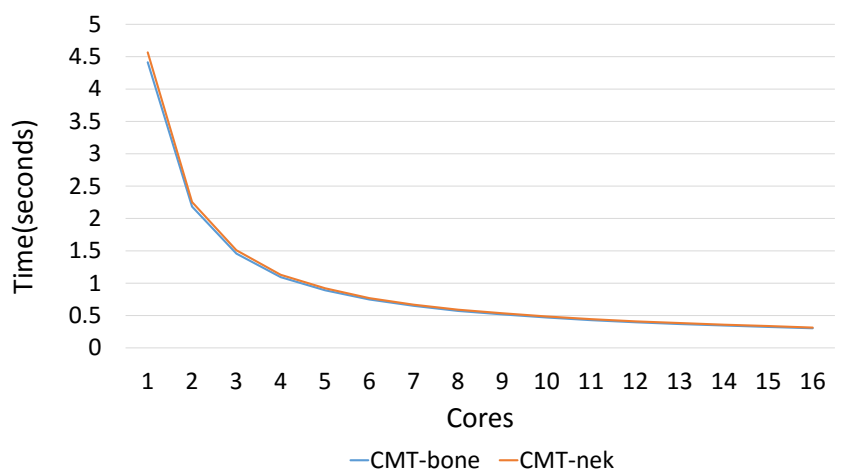

Fig. 12: Comparison of run times of CMT-nek and CMT-bone for the compute_kernel.

computation to data access ratio.

Qbox [16] is a first-principle molecular dynamics code that is used to compute properties of materials. A Qbox run stresses memory bandwidth, is characterized by high floating-point intensity and uses global collectives. The two main computational operations are dense linear algebra implemented in a parallel fashion using ScaLAPACK library, and a custom 3D Fast Fourier Transform. The primary data structure is the elec- 


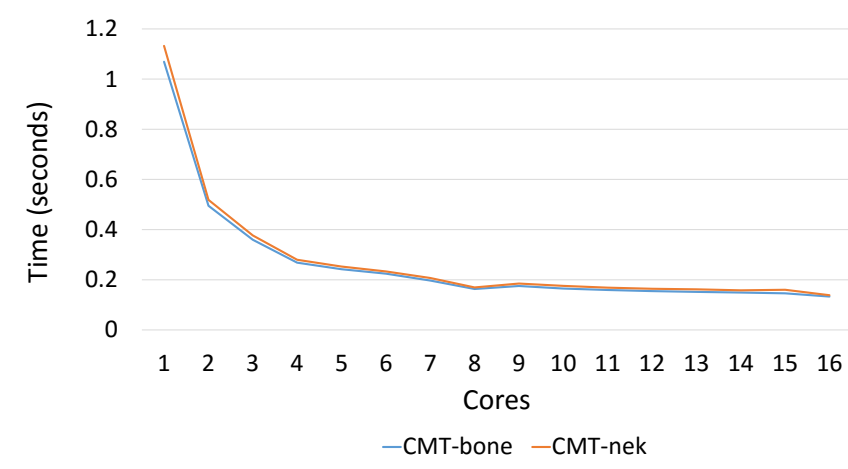

Fig. 13: Comparison of run times of CMT-nek and CMT-bone for the comm_kernel.

tronic wavefunction that is distributed across MPI processes. The main computation operation are matrix multiplication, Gram-Schmidt orthogonal operations and parallel FFTs.

Validating proxy application is as important as developing one. Islam et al. analyzes validation methodologies in [3], as their development validates parent application OPENMC and its proxy XSBENCH. OPENMC uses Monte Carlo simulations to compute the path of particle neutrons through a nuclear reactor using Monte Carlo simulations. The proxy XSBENCH represents the most compute intensive kernels of OPENMC which requires about $85 \%$ of the total runtime of OPENMC.

Development of CMT-bone will allow us to solve complex problems such as code implementation and designing dynamic load balancing algorithms on hybrid platforms. Particle movement and varying density of particles make load balancing an important feature in CMT-nek. Efficient data decomposition is required while taking into consideration the different capacities of the processing elements in a hybrid platform. The methods in [17] show how particles and spectral elements may be mapped in a simple architecture independent representation, such as a one dimensional array, while still preserving node locality. The methods in [18] present optimization for fast mapping on hybrid architectures, while methods in [19] show how to map the many-to-many communication generated.

\section{CONCLUSIONS}

In this paper, we describe a new proxy-application called CMT-bone. CMT-bone has key implementation components of CMT-nek and has several memory structures for storing volume data, surface data and particle data. CMT-bone was validated to be a representative application of CMT-nek using VERITAS.

The coupling between the different data structures, along with computation and communication structures that are representative of other large scale CFD application make it an interesting application for evaluating a variety of parallel architectures in terms of performance, scalability and communication bottlenecks. Additionally, the dynamic nature of the computational load due to particle simulation in CMT-bone should also allow for the study of dynamic load balancing algorithms.
We demonstrated the validation of the proxy app and parent app using validation procedure using VERITAS [3]. The experimental results demonstrated that the proxy app is a good representative of the parent application. Additionally areas of potential difference were also highlighted.

\section{ACKNOWLEDGMENT}

This work was funded by the U.S. Department of Energy, National Nuclear Security Administration, Advanced Simulation and Computing Program, as a Cooperative Agreement under the Predictive Science Academic Alliance Program, Contract No. DOE-NA0002378.

\section{REFERENCES}

[1] P. F. Fischer, J. Lottes, S. Kerkemeier, K. Heisey, A. Obabko, O. Marin, and E. Merzari. http://nek5000.mcs.anl.gov, 2014.

[2] P.F. Fischer H.M. Tufo. Terascale spectral element algorithms and implementations. In In Proceedings of the 1999 ACM/IEEE conference on Supercomputing, page 68. ACM, 1999.

[3] Tanzima Zerin Islam, Jayaraman J. Thiagarajan, Abhinav Bhatele, Martin Schulz, and Todd Gamblin. A machine-learning framework for performance coverage analysis of proxy applications. In International Conference for High Performance Computing, Networking, Storage and Analysis (SC), 2016.

[4] S. Gottlieb and C.-W. Shu. Total variation-diminishing Runge-Kutta schemes. Math. Comp., 67:73-85, 1998.

[5] M. S. Liou. A sequel to AUSM: AUSM+. J. Comp. Phys., 129:362-382, 1996.

[6] M. Parmar, A. Haselbacher, and S. Balachandar. Generalized BassetBoussinesq-Oseen equation for unsteady forces on a sphere in a compressible flow. Phys. Rev. Lett., 106:084501, 2011.

[7] M. O. Deville, P. F. Fischer, and E.H. Mund. High-order methods for incompressible fluid flow. Cambridge University Press, Cambridge, 2002.

[8] J. S. Hesthaven and T. Warburton. Nodal discontinuous Galerkin methods: Algorithms, analysis, and applications. Springer, New York, 2008.

[9] C. Canuto, M. Y. Hussaini, A. Quarteroni, and T.A. Zang. Spectral Methods I: Fundamentals in single domains. Springer-Verlag, New York, 2006.

[10] B. Fornberg. A practical guide to pseudospectral methods. Cambridge University Press, New York, 1998.

[11] R. M. Kirby and G. E. Karniadakis. De-aliasing on non-uniform grids: algorithms and applications. J. Comp. Phys., 191:249-264, 2003.

[12] A.M. Bruckstein, Michael Elad, and Michael Zibulevsky. On the uniqueness of nonnegative sparse solutions to underdetermined systems of equations. IEEE Transactions on Information Theory, 54(11):48134820, 2008.

[13] Coral benchmark codes. https://asc.1lnl.gov/CORAL-benchmarks, 2014.

[14] Proxy-apps for thermal hydraulics. https://cesar.mcs.anl.gov/content/ software/thermal_hydraulics.

[15] Co-design at lawrence livermore national lab. https://codesign.llnl.gov/ amg2013.php.

[16] Qbox qb@1l branch. https://asc.llnl.gov/CORAL-benchmarks/ Summaries/QBox_Summary_v1.2.pdf.

[17] Chao-Wei Ou, Manoj Gunwani, and Sanjay Ranka. Architectureindependent locality-improving transformations of computational graphs embedded in k-dimensions. In Proceedings of the 9th International Conference on Supercomputing, ICS '95, pages 289-298, New York, NY, USA, 1995. ACM.

[18] Maher Kaddoura, Chao-Wei Ou, and Sanjay Ranka. Runtime support for parallelization of data-parallel applications on adaptive and nonuniform computational environments. Journal of Parallel and Distributed Computing, 43:163-168, 1997.

[19] Sanjay Ranka, Ravi Shankar, and Khaled Alsabti. Many-to-many personalized communication with bounded traffic. In Frontiers of Massively Parallel Computation, Frontiers' 95, pages 20-27, 1995. 